\title{
DNA methylation profile of Aire-deficient mouse medullary thymic epithelial cells
}

\author{
Guoying Wu', Keiji Hirabayashi', Shinya Sato ${ }^{1}$, Nobuko Akiyama², Taishin Akiyama², Kunio Shiota \\ and Shintaro Yagi ${ }^{*}$
}

\begin{abstract}
Background: Medullary thymic epithelial cells (mTECs) are characterized by ectopic expression of self-antigens during the establishment of central tolerance. The autoimmune regulator (Aire), which is specifically expressed in mTECs, is responsible for the expression of a large repertoire of tissue-restricted antigens (TRAs) and plays a role in the development of mTECs. However, Aire-deficient mTECs still express TRAs. Moreover, a subset of mTECs, which are considered to be at a stage of terminal differentiation, exists in the Aire-deficient thymus. The phenotype of a specific cell type in a multicellular organism is governed by the epigenetic regulation system. DNA methylation modification is an important component of this system. Every cell or tissue type displays a DNA methylation profile, consisting of tissue-dependent and differentially methylated regions (T-DMRs), and this profile is involved in cell-type-specific genome usage. The aim of this study was to examine the DNA methylation profile of mTECs by using Aire-deficient mTECs as a model.

Results: We identified the T-DMRs of mTECs (mTEC-T-DMRs) via genome-wide DNA methylation analysis of Aire ${ }^{-/-}$ mTECs by comparison with the liver, brain, thymus, and embryonic stem cells. The hypomethylated mTEC-T-DMRs in Aire ${ }^{-1-}$ mTECs were associated with mTEC-specific genes, including Aire, CD80, and Trp63, as well as other genes involved in the RANK signaling pathway. While these mTEC-T-DMRs were also hypomethylated in Aire ${ }^{+/+}$mTECs, they were hypermethylated in control thymic stromal cells. We compared the pattern of DNA methylation levels at a total of 55 mTEC-T-DMRs and adjacent regions and found that the DNA methylation status was similar for Aire ${ }^{+/+}$ and Aire ${ }^{-/-}$mTECs but distinct from that of athymic cells and tissues.

Conclusions: These results indicate a unique DNA methylation profile that is independent of Aire in mTECs. This profile is distinct from other cell types in the thymic microenvironment and is indicated to be involved in the differentiation of the MTEC lineage.
\end{abstract}

Keywords: Medullary thymic epithelial cells, Aire, T-DMR

\section{Background}

Medullary thymic epithelial cells (mTECs) are highly involved in the establishment of central tolerance by ectopically expressing a variety of tissue-restricted antigens (TRAs) [1]. Deficiency of the autoimmune regulator, Aire-which regulates a large pool of genes in mTECs-results in autoimmune polyglandular syndrome in humans and autoimmunity in mice [2]. There are

\footnotetext{
* Correspondence: asyagi@mail.ecc.u-tokyo.ac.jp

'Laboratory of Cellular Biochemistry, Department of Animal Resource

Sciences Neterinary Medical Science, Graduate School of Agricultural and

Life Sciences, The University of Tokyo, 1-1-1, Yayoi, Bunkyo-ku, Tokyo

113-8657, Japan

Full list of author information is available at the end of the article
}

estimated to be hundreds to over one thousand TRAs that are activated by Aire in mTECs, based on microarray data from the transcriptomes of wild-type and Aire-deficient mTECs [3,4]. However, numerous TRAs are expressed in mature mTECs in an Aire-independent pattern [3,4]. Moreover, single-cell PCR analysis with sorted mTECs demonstrated that approximately 70\% [5], or even fewer [6], of mature mTECs express Aire, and TRAs are expressed in Aire-negative mTECs [5]. These findings indicate that Aire is necessary, but not sufficient, for the expression of TRAs in mTECs.

The role of Aire in the maturation of mTECs has been indicated by using Aire-deficient mice, which exhibit contracted compartments in the thymic medulla and an

\section{Biomed Central}


increased number of medullary cells positive for p63, whose expression is diminished in Aire-positive mTECs [6-9]. The role of Aire in the differentiation of mTECs has been reviewed by Matsumoto [10]. However, the existence of the medullary compartment and the increased number of a small subset of $\mathrm{K}^{-} \mathrm{K}^{+}$cells-considered to represent end-stage and terminally differentiated mTECs -in the Aire-deficient thymus indicate a limited role of Aire in the lineage determination of mTECs, in which the RANK signaling pathway is involved $[11,12]$. Thus, Aire-deficient mTECs may represent a suitable model to study not only Aire-independent TRA expression but also the fundamental regulation system that governs cell lineage determination of mTECs.

Genome-wide analysis of DNA methylation has indicated that a specific DNA methylation profile exists for every cell or tissue type. This profile consists of numerous tissue-dependent and differentially methylated regions (T-DMRs) at cytosine residues in CpG dinucleotides (CpGs), which are differentially methylated among distinct cells and tissues [13]. DNA methylation profiles change during cell differentiation, reflect developmental similarity among cell lineages [14,15], and illuminate cell- or tissue-specific gene expression profiles [16-18]. These findings suggest that DNA methylation profiles are involved in the establishment and maintenance of the regulatory system for cell- or tissue-type-specific genome use, and could therefore be used to identify types of cells and tissues.

The DNA methylation profile responsible for the phenotype of mTECs is still largely unknown, although a limited number of genes, including Aire and 2 Aireindependent TRA genes, Csn2 (Csnb) and Gad1 (Gad67), are unmethylated in their promoter regions [19-21]. To explore the genome-wide DNA methylation of mTECs, we applied a microarray-based screening system, T-DMR profiling with restriction-tag-mediated amplification (D-REAM) [16], to UEA1 ${ }^{+} \mathrm{CD} 45^{-}$mTECs that were isolated from Aire-deficient $\left(\right.$ Air $\left.^{-1-}\right)$ mice [22]. We identified T-DMRs in mTECs (mTEC-T-DMRs) and compared the DNA methylation status at mTEC-TDMRs in $A i r e^{+/+}$and Aire ${ }^{-/-}$mTECs, UEA1 ${ }^{-} \mathrm{CD} 45^{-}$ thymic stromal cells, and athymic cell and tissues.

\section{Results and discussion}

\section{mTEC-T-DMRs identified in Aire ${ }^{-/-}$mTECs are associated} with $\mathrm{mTEC}$-specific genes

Thymic stromal cells from Aire $e^{-/-}$mice and their Aire $e^{+/+}$ littermates were fractioned into 2 populations: UEA1 ${ }^{+} \mathrm{CD} 45^{-}$(mTECs) and UEA $1^{-} \mathrm{CD} 45^{-}$(control thymic stromal cells). In this study, we use the term "stromal" to refer to UEA1 ${ }^{-} \mathrm{CD} 45^{-}$cells, which are a mixture of keratinexpressing epithelial cells, including cortical TECs (cTECs), and keratin-negative mesenchymal cells such as fibroblasts, connective tissue cells, and endothelial cells [23]. Predominant expression of Aire, Cd80, Krt5, Krt8, Krt14, and Foxn1 in the UEA $1^{+} \mathrm{CD} 45^{-}$population (detected by RTPCR) indicated enrichment of mTECs in this fraction (Additional file 1: Figure S1). To elucidate the characteristics of genome-wide DNA methylation in mTECs, we compared Aire ${ }^{-/-}$mTECs with the liver, brain, thymus, and embryonic stem (ES) cells by D-REAM. mTEC-T-DMRs, which were differentially methylated as compared with the other cells and tissue types, were screened as genomic fragments that exhibited significantly different microarray scores after digestion by the methylation-sensitive restriction enzyme, HpyCH4IV (Additional file 2: Figure S2). Among the mTEChypo-T-DMRs, which were hypomethylated in Aire $^{-/}$mTECs, we designated those that exhibited scores higher than all or any 2 of the reference cells and tissues as mTEC-unique T-DMRs (mTECu-T-DMRs) or mTECany2-T-DMRs, respectively (Figure 1A, Additional file 3: Table S1).

The mTECany2-T-DMRs mapped to within $8.5-\mathrm{kb}$ regions around the transcription start sites (TSSs) of 3081 ENSTs (Ensembl transcripts; mm9) (Additional file 3: Table S1). The distribution of mTECany2-T-DMRs exhibited a similar pattern to that observed in previous analyses of somatic tissues and ES cells $[16,17]$, displaying a peak of T-DMRs at the 3'-downstream regions of TSSs (Additional file 4: Figure S3). Hypomethylated signals in these regions are often associated with cell- and tissue-specific highly expressed genes [16,17].

We analyzed the expression of ENSTs associated with mTECany2-T-DMRs in mTECs using the gene expression profile of CDR $1^{\text {int }} \mathrm{B} 7-1^{\text {hi }}$ CD $45^{-}$mTECs from the Gene Expression Omnibus (GEO) database, and compared with those of mouse liver, and $\mathrm{CD} 4{ }^{+} \mathrm{CD} 8^{+}$thymocytes as a representative of the thymic cells (Additional file 5: Table S2). The differences in expression levels of genes associated with mTECany2-T-DMRs were relatively small between Aire $^{-/-}$and Aire ${ }^{+/+}$mTECs as compared with those between mTECs and the liver or thymocytes, indicating that the expression patterns of genes associated with mTECany2-T-DMRs in mTECs are not affected by functional Aire (Additional file 6: Figure S4). High gene expression in mTECs was more prominent for genes associated with mTECany2-T-DMRs in the $-1.5 \mathrm{~kb}$ upstream and $+2.5 \mathrm{~kb}$ downstream (proximal) regions of TSSs. Among these genes, those associated with proximal mTECu-TDMRs showed significantly higher expression in mTECs compared with both the liver and thymocytes (Figure 1B). These data indicate that mTEC-specific genes are likely to be associated with mTECu-T-DMRs, and methylation may be responsible for gene silencing in Aire non-expressing tissues and cells. Indeed, we found that among the mTECu-T-DMR-associated genes, some had been previously reported as mTEC-specific genes, of which the 
majority showed Aire-independent expression in mTECs (Additional file 7: Table S3). Moreover, marker genes of mTECs, including Aire, $C d 80$, and Trp63, were found to be associated with proximal mTECu-T-DMRs.
We analyzed the DNA methylation status around the mTECu-T-DMR of Aire which is located downstream of its TSS, and the upstream region by using bisulfite sequencing (Figure $1 \mathrm{C}$ ). In the -624 to -218 bp upstream region, the

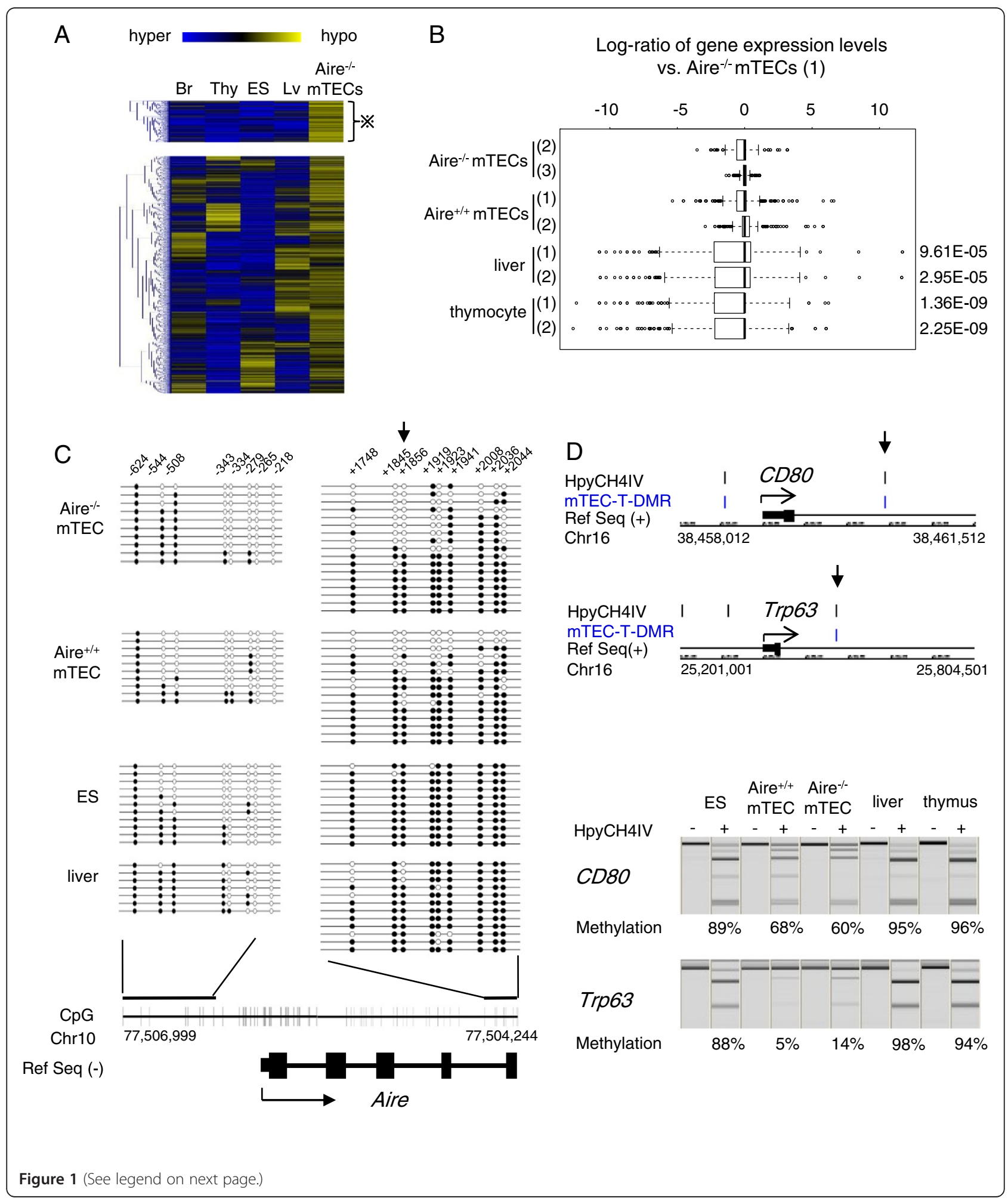


(See figure on previous page.)

Figure 1 mTECany2-T-DMRs correlate with gene expression in mTECs and associate with marker genes of mTECs. A. Hierarchical clustering of D-REAM scores corresponding to mTECany2-T-DMRs identified by comparison of Aire ${ }^{-/-}$mTECs with the brain, thymus, liver, and ES cells by Pearson's correlations. Among mTECany2-T-DMRs, those hypomethylated in Aire ${ }^{-/-}$mTECs in comparison with all of the reference cells and tissues were defined as mTECu-T-DMRs (indicated by asterisk). Br, brain; Thy, thymus; Lv, liver. B. Relative expression levels of genes associated with mTECu-T-DMRs in proximal regions $(-6$ to $+2.5 \mathrm{~kb})$ from TSS. The horizontal axis of the boxplot represents the log ratios of gene expression levels relative to Aire ${ }^{-1-}$ mTECs. The numbers in round brackets indicate independent gene expression profiles for each type of cell or tissue (Additional file 5: Table S2). The $p$-values of $t$-tests showing significant differences in relative expression levels are shown. $\mathbf{C}$. Bisulfite sequencing of regions around the TSS of Aire. The arrowhead indicates the position of the mTECu-T-DMR. The regions upstream (-674 to -191 bp) and downstream (+1672 to +2082 bp) of the TSS were analyzed independently using bisulfite sequencing. Open and closed circles represent unmethylated and methylated CpGs, respectively. Positions of CpGs are shown as vertical bars above the genomic structure (shown at the bottom of the panel), and the boxes indicate exons. D. COBRA analysis of mTECu-T-DMRs associated with Cd80 and Trp63. Positions of HpyCH4IV sites and mTECU-T-DMRs are shown above the genomic sequences within -1 to $+2.5 \mathrm{~kb}$ of the TSS. Electropherograms represent the bisulfite PCR products after digestion with HpyCH4IV. Hypomethylated fragments were resistant to HpyCH4IV digestion (+). The levels of DNA methylation (\%) are shown under each lane of the electropherograms.

liver, which represents an Aire non-expressing tissue, showed a methylated status comparable with Aire $^{+/+}$and Aire $^{-/-}$mTECs as well as with ES cells, which express Aire [24]. The promoter regions of human AIRE ( -295 to +65 bp) and mouse Aire ( -287 to $+133 \mathrm{bp})$ are both associated with a CpG island ( $>50 \%$ CpG content) and are unmethylated in mTECs $[19,20]$. Since Aire-negative cTECs and several extrathymic tissues were also found to be unmethylated at this region [20], hypomethylation of the promoter is considered to be necessary, but not sufficient, for expression. In contrast, Aire ${ }^{+/+}$and Aire ${ }^{-/-}$mTECs, but not ES cells, exhibited a hypomethylated status at the 9 CpGs in the region between +2082 and +1672 bp from the TSS, including the CpG corresponding to the mTECu-T-DMR. Among the tissue-specific genes carrying CpG islands within $1 \mathrm{~kb}$ of their TSSs, hypomethylated T-DMRs tend to be located downstream of TSSs $[16,17]$. Thus, the mTECTDMR for Aire located 3'-downstream of the Aire TSS could serve as an epigenetic marker to distinguish mTECs from Aire-expressing ES cells, in which the expression level of Aire was significantly less than that in the thymus and mTECs (Additional file 1: Figure S1).

The mTECu-T-DMRs associated with CD80 and p63 (Trp63) were analyzed with combined bisulfite restriction analysis (COBRA). Both Aire And Aire $^{-/-}$mTECs exhibited a hypomethylated status compared with the liver, thymus, and ES cells (Figure 1D). Notably, the mTECu-T-DMR of p63 was found to be associated with $\triangle \mathrm{Np} 63$, the short isoform of p63, which is essential for maintenance of the progenitor status of thymic epithelial cells [25] and not expressed in Aire-positive mTECs [79]. Hypomethylation at this T-DMR in Aire ${ }^{+/+}$mTECs suggests that DNA methylation does not determine the repression of p63 in Aire ${ }^{+/+}$mTEC cells.

mTECany2-T-DMRs are specific to mTECs, as compared with the other thymic stromal cells

We compared the DNA methylation levels of 15 selected genes-which were shown to be associated with
mTECany2-T-DMRs-between the mTECs and stromal cells from Aire A $^{+/+}$and Aire $e^{-/-}$mice by COBRA (Figure 2). In contrast to the hypomethylated status in mTECs, Aire, Cd80, and Trp63 were hypermethylated in stromal cells. Some genes that associated with mTECany2-T-DMRs were found to be involved in the RANK/RANKL signaling pathway, which stimulates the downstream NF-kB pathway and is required for the development of mTECs [11,12,26] (Additional file 8: Figure S5). For example, Tnfrsf11a (RANK) was hypomethylated in mTECs but hypermethylated in stromal cells. Traf2, the gene downstream of RANK, was hypomethylated not only in mTECs but also in the stroma, while its downstream genes, Tank, Birc2, and Edaradd demonstrated similar methylation patterns to Tnfrsf11a. We also found that H2-Dma (H2-Dm), which exhibited 2 mTECu-T-DMRs surrounding its second exon-a region essential for antigen presentation by MHCII [27]—was hypomethylated in mTECs and hypermethylated in the remaining stromal cells. In addition to these genes, similar methylation patterns were observed at mTECany2-T-DMRs associated with genes exhibiting tissue-specific expression patterns, such as Aplp2 (adipose tissue and brain), Arg1 (liver), Ctla4 (Treg), Ehhadh (kidney and liver), Mtap7 (lens), and Slc36a3 (testis). Importantly, these methylation patterns were observed in both Aire $^{-/-}$and Aire ${ }^{+/+}$mTECs, demonstrating that a DNA methylation pattern distinct from other thymic stromal cells is maintained in Aire $^{+/+}$ mTECs.

\section{Aire $^{+/+}$and Aire ${ }^{-/-}$mTECs show similar DNA methylation levels at $\mathrm{mTEC}-\mathrm{T}-\mathrm{DMRs}$}

To further compare the DNA methylation status between Aire $^{+/+}$and Aire ${ }^{-/-}$mTECs, we used COBRA to analyze 55 loci, including mTEC-T-DMRs and HpyCH4IV sites in the vicinity and some previously identified T-DMRs of other cell and tissue types [16,17] (Figure 3, Additional file 9: Table S4). Hierarchical clustering of DNA methylation levels at these mTEC-T-DMRs showed that Aire $^{+/+}$ 

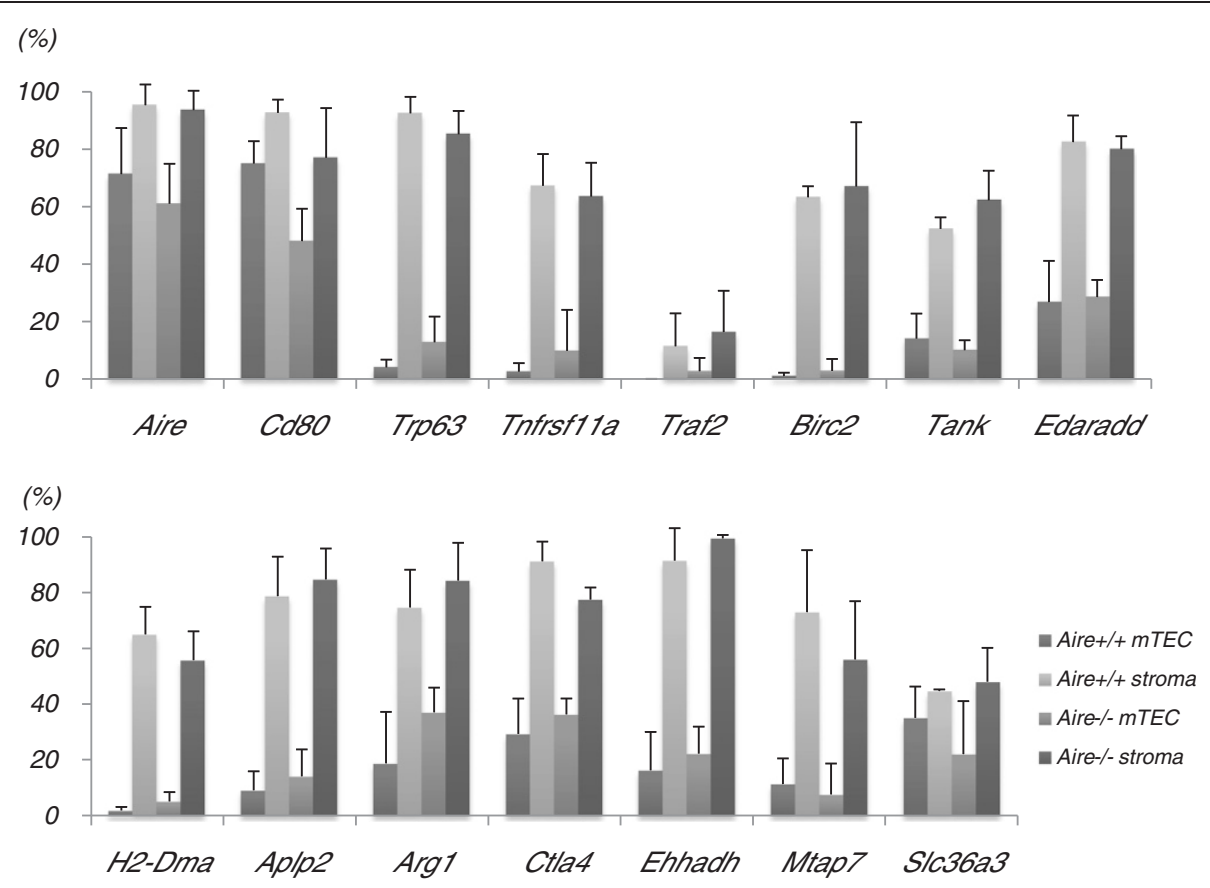

Figure 2 UEA $1^{+} \mathrm{CD} 45^{-} \mathrm{mTECs}$ and UEA $1^{-} \mathrm{CD} 45^{-}$thymic stromal cells display distinct DNA methylation levels at mTECany2-T-DMRs.

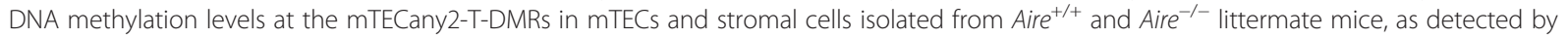
COBRA. The levels of DNA methylation at CpGs within the HpyCH4IV site are indicated by the vertical axis. For Aire and CD80 genes—containing $2 \mathrm{HpyCH} 4 \mathrm{IV}$ sites inside the investigated region - the DNA methylation rate was calculated when any 1 of the CpGs was methylated. For the remaining genes, the levels represent DNA methylation levels at a single CpG. Bar graphs indicate means \pm standard errors of the data from at least 3 independent bisulfite PCRs using independently isolated cells from 3 Aire ${ }^{+/+}$mice and 3 Aire ${ }^{-/-}$mice.

and Aire $^{-1-}$ mTECs display the most similar pattern (less than $20 \%$ difference in DNA methylation) compared with the liver, thymus, and ES cells. DNA methylation levels at mTEChypo-T-DMRs in both mTECs showed a unique or common hypomethylated status compared with the reference cell and tissues (Figure 3, upper panel), as revealed by D-REAM analysis (Figure 1A). Genes associated with TDMRs characteristic of other cell and tissues were hypermethylated in mTECs (Figure 3. lower panel). These data indicate that the presence of Aire does not greatly change DNA methylation levels in mTECs.

Among the analyzed genes, Fgg (fibrinogen gamma chain), which is a liver-specific gene that is expressed in mTECs in an Aire-dependent pattern [3,4], was hypermethylated in both Aire ${ }^{+/+}$and Aire ${ }^{-/-}$mTECs, similar to its status in ES cells and the thymus. The gene expression of TRAs in mTECs occurs in specific subpopulations and in a stochastic manner [1,2]; therefore, DNA methylation changes may occur at specific loci in an Aire-dependent manner in subpopulations of mTECs that express a specific TRA.

During thymic organogenesis, Aire is expressed at E16.5 [28], while the stromal cells of the medulla and cortex are separated at embryonic day 13 (E13) [29]. In the absence of Aire, mTECs survive Aire-induced apoptosis, reach a terminally differentiated stage [10], and maintain the ability to express TRAs [3,4]. Thus, the similar DNA methylation status at T-DMRs in Aire $^{+/+}$ and Aire ${ }^{-1-}$ mTECs suggests the existence of an Aireindependent DNA methylation profile that serves as an intrinsic system to determine the phenotype of mTECs.

\section{Conclusions}

In this study, we demonstrated the DNA methylation profile for Aire $^{-l-}$ mTECs by genome-wide identification of T-DMRs. The DNA methylation status at dozens of mTEC-T-DMRs in Aire ${ }^{+/+}$mTECs was similar to that in Aire $^{-/-}$mTECs, while distinct from athymic cells and tissues as well as the other thymic stromal cells, indicating that the Aire-independent DNA methylation profile is involved in differentiation of the MTEC lineage and other thymic stroma cells that form the thymic microenvironment.

\section{Methods}

Mice

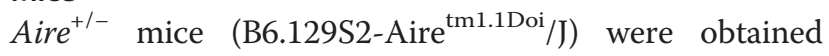
from the Jackson Laboratory [22] and backcrossed onto 


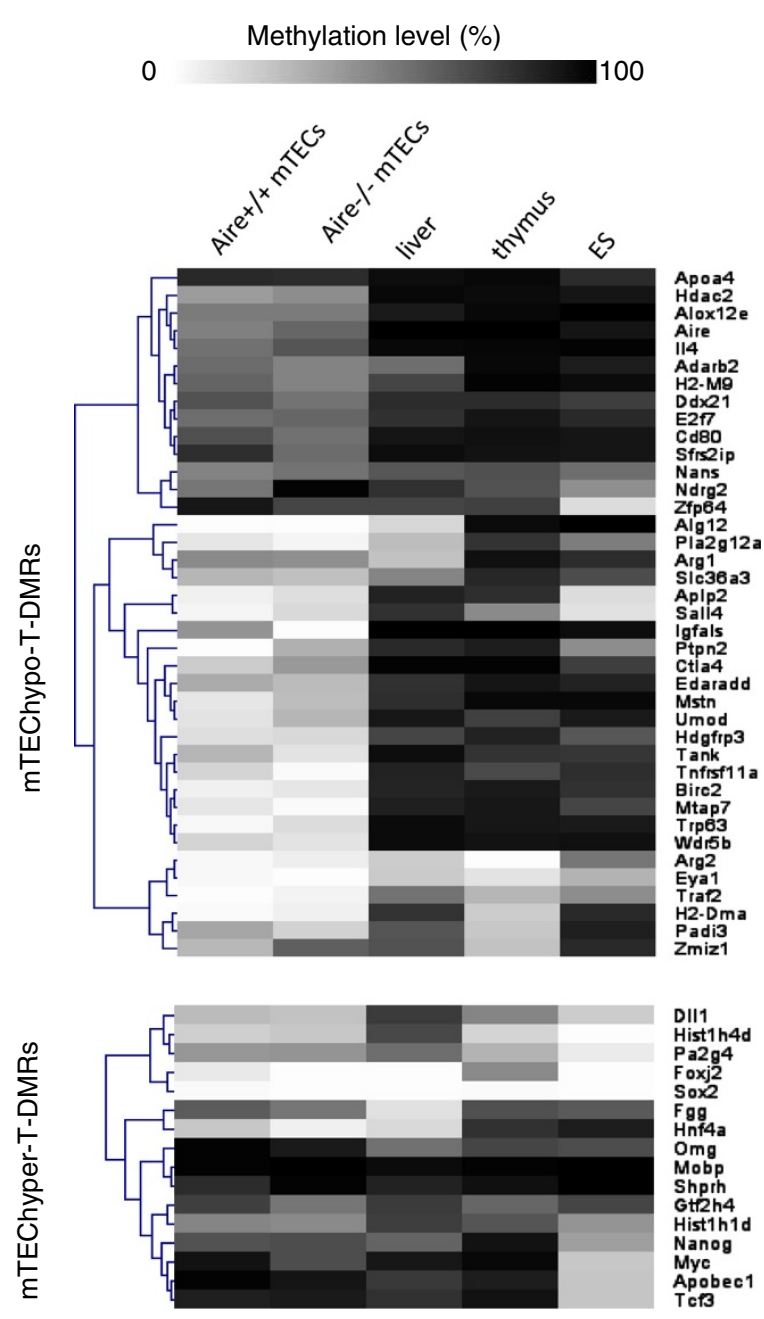

Figure 3 Aire $^{+/+}$and Aire ${ }^{-/-}$mTECs show similar DNA methylation profiles. Hierarchical clustering with Euclidean distance of DNA methylation levels estimated by COBRA at 39 mTECany2-T-DMRs and adjacent T-DMRs (upper panel) and 16 previously identified tissue T-DMRs [16,17], which overlap with mTEChyper-T-DMRs (lower panel, Additional file 3: Table S1). DNA methylation levels from 0\% to 100\% are represented as the continuous gradation from white to black. Each row represents a different gene associated with T-DMRs.

C57BL/6J (B6) mice (Oriental Yeast, Japan). Aire ${ }^{-/-}$mice and their Aire $^{+/+}$littermates were used for isolation of mTECs. The mice were housed and bred under specific pathogen-free conditions.

All experiments using mice were carried out according to the institutional guidelines for the care and use of laboratory animals (Graduate School of Agricultural and Life Sciences, the University of Tokyo).

\section{Isolation of mTECs}

The isolation of mTECs was conducted by modifying previously described methods [16,30]. Briefly, thymi were collected from 6- to 7-week-old male mice, finely minced, washed in RPMI 1640 medium, and sequentially digested twice with $0.2 \mathrm{mg} / \mathrm{mL}$ collagenase $\mathrm{D}$ for $15 \mathrm{~min}$ at $37^{\circ} \mathrm{C}$. The pellets were incubated with $0.2 \mathrm{mg} / \mathrm{mL}$ collagenase D and $0.2 \mathrm{mg} / \mathrm{mL}$ Dispase I (Roche Applied Science) with $25 \mu \mathrm{g} / \mathrm{mL}$ DNase I (Takara), and the reaction was stopped with $5 \mathrm{mM}$ EDTA. Subsequently, after centrifugation at $1350 \times g$ on a Percoll gradient, supernatants between $\rho=1.06 \mathrm{~g} / \mathrm{mL}$ and $\rho=1.0 \mathrm{~g} / \mathrm{mL}$ were incubated with Mouse BD Fc Block (2.4G2), PE Rat Anti-Mouse CD45 (30-F11) (BD Pharmingen), and FITC-conjugated UEA-1 (Sigma) and sorted using an EPICS Altra flow cytometer (Beckman-Coulter). The fractionation of cells was monitored by RT-PCR (Additional file 10).

\section{D-REAM analysis}

In D-REAM analysis, differential methylation status at HpyCH4IV loci is indicated by differential scores corresponding 
to fragments generated by this methylation-sensitive restriction enzyme between 2 samples [16; Additional file 2: Figure S2]. In the present study, we performed microarray experiments using mTECs from Aire ${ }^{-/-}$mice and the thymus from C57BL/6J mice. Briefly, after digestion of genomic DNA with HpyCH4IV (New England Biolabs), $50 \mathrm{ng}$ of DNA was ligated with an adaptor, followed by TaqI digestion and ligation with a second adaptor. The fragments were amplified by ligationmediated PCR to allow selective amplification of unmethylated fragments generated by HpyCH4IV digestion and then hybridized using the GeneChip Mouse Promoter 1.0R Array (Affymetrix), which comprises probes that map to the regions $(-6$ to $+2.5 \mathrm{~kb})$ around the TSSs of approximately 50,000 transcripts (ENST IDs; mm9) obtained from the UCSC genome browser. Hybridization signals were processed with MAT [31] and can be found at Array Express (http://www.ebi. ac.uk/arrayexpress/; accession number: E-MTAB-1113). The data from Aire ${ }^{-/-}$mTECs and the thymus were compared with D-REAM data from the liver, brain, and ES cells [17] to identify fragments that exhibited a difference in microarray scores, thereby indicating the DNA methylation status at corresponding HpyCH4IV sites. We set the cut-off value at $0.5 \%$ of FDR (false detecting rates) for T-DMRs. The Galaxy genome browser (https://main.g2.bx.psu.edu/) [32] and "R" (http://www.R-project.org) [33] were used for genomic fragment analysis.

To analyze the gene expression of Aire $^{+/+}$and Aire mTECs, we obtained their gene expression profiles from the GEO database (http://www.ncbi.nlm.nih.gov/geo/) [34] and compared with those of the liver and $\mathrm{CD} 4^{+} \mathrm{CD} 8^{+}$ thymocytes on the same microarray platform (Additional file 5: Table S2). The array data were normalized using the gcrma package [35]. The ENST IDs were converted into Affymetrix IDs by using BioMart software (http://www. biomart.org) [36].

\section{DNA methylation analysis with bisulfite-converted genomic DNA}

mTEC cells and gDNAs from the thymus, liver, and ES cells were subjected to bisulfite treatment using the EZ DNA Methylation-Direct Kit (Zymo Research Cooperation) according to the manufacturer's instructions. Unmethylated cytosine residues are converted into thymine residues by the sodium bisulfite reaction, while methylated cytosine residues remain unchanged. PCR was carried out using the bisulfite-converted genomic DNA with the primers listed in Additional file 11: Table S5. To evaluate the DNA methylation status at specific CpGs, COBRA was performed. The PCR products were evenly divided and incubated with or without HpyCH4IV for $5 \mathrm{~h}$ at $37^{\circ} \mathrm{C}$ and then analyzed with a microchip electrophoresis system (MultiNA, Shimadzu Biotech). DNA methylation levels were calculated as the percentage of digested fragment compared with the sum of digested and undigested fragments. To evaluate the DNA methylation status at CpGs within hundreds of base pairs, the PCR fragments were cloned into the pGEM-T Easy Vector (Promega), and more than 10 clones were sequenced using an $\mathrm{ABI}$ 3130 sequencer with a BigDye Terminator kit (Applied Biosystems).

\section{Additional files}

\begin{abstract}
Additional file 1: Figure S1. Isolation and characterization of CD45 UEA $1^{+}$mTECs and CD45 UEA $1^{-}$thymic stromal cells. Isolation and characterization of CD45 UEA $1^{+}$mTECs and CD45 UEA $1^{-}$thymic stromal cells. A. Isolation of CD45 UEA $1^{+}$mTECs (red) and CD45 UEA thymic stromal cells (yellow) by flow cytometry. Values are representatives of the percentage of each population in repeated isolations. B. Gene expression in mTECs, thymic stromal cells, thymus, liver and ES cells by RT-PCR. Compared with the stromal cells, higher expressions of Aire, Cd80, Krt5, Krt14, Krt8, Foxn 1 in both the Aire ${ }^{+/+}$and Aire ${ }^{-/-}$mTECs were detected. The Aire $^{-1-}$ mice strain used in this research lacks the exon2 of Aire, but the transcription of Aire could be detected [1]. E2, exon2; E3, exon3; E5, exon5. C. Real-time RT-PCR quantification of transcript levels of Aire in mTECs isolated from Aire ${ }^{+/+}$mice, as well as mTEC, thymus from C57BL/6 strain, and ES cells. The Exon2 to Exon3 of Aire mRNA was amplified. Standard curve method calculation was used to compute transcript levels relative to $A c t b$ mRNA. The y axis indicates arbitrary unit normalized to Actb, with the expression level in thymus as 1 . The expression level of Aire in mTECs was over 50 times higher than in the thymus, which showed a similar pattern as human AIRE in MTECs and the thymus [2]. The expression level of Aire in ES was about half of the thymus. Expression of Aire in liver was not detected.
\end{abstract}

Additional file 2: Figure S2. Illustration of the D-REAM method for identification of T-DMRs. D-REAM was developed via integration of a methylation-sensitive restriction enzyme ( $\mathrm{HpyCH} 4 \mathrm{IV})$, ligation-mediated PCR (LM-PCR), and DNA tiling microarrays [3,4]. Briefly, for a certain genomic locus that contains a HpyCH4IV recognizing site (ACGT), in Sample A, the ACGT containing 5/-methylcytosine cannot be digested by HpyCH4IV and the corresponding fragment is not amplified by LM-PCR, thus does not detected by microarray. In contrast, in Sample B, the ACGT with unmethylated cytocine is digested and the corresponding fragment is efficiently amplified, leading to microarray signal, reflected by D-REAM score. Comparing the two samples using the D-REAM score, the locus is defined as a T-DMR and hypomethylated in Sample B.

Additional file 3: Table S1. Summary of mTEC-T-DMRs.

Additional file 4: Figure S3. Distributions of positions relative to TSS of mTECany2-T-DMRs and mTECu-T-DMRs. The highest frequencies of T-DMRs are in the 3 l downstream to TSSs in both mTECany2-T-DMRs and mTECu-T-DMRs. For the histogram, on unit represents $250 \mathrm{bp}$.

Additional file 5: Table S2. List of microarray data of transcriptomes used in this study.

Additional file 6: Figure S4. Log ratio of the expression levels of genes associated the mTECu-T-DMRs and mTECany2-T-DMRs. Relative expression levels of genes associated with mTECU-T-DMRs (upper) and mTECany2-T-DMRs (lower) in Aire ${ }^{-/-}$mTECs, Aire ${ }^{+/+}$mTECs, liver and CD4 ${ }^{+} \mathrm{CD} 8{ }^{+}$thymocytes to Aire ${ }^{-1-}$ mTECs. From left to right, the data sets represented the all probed genes and the T-DMRs, classified according to their relative distance to TSSs as -6 to $+2.5 \mathrm{~kb}$ (whole window), -1 to $+2.5 \mathrm{~kb}$ (proximal) and -6 to $-1 \mathrm{~kb}$ (distal) regions. The vertical axis of the box-plot represents the values of gene expression levels of duplicated data sets relative to one microarray profile of Aire ${ }^{-/-}$mTECs. The numbers in the round brackets indicate independent gene expression profiles of each type of cell or tissues listed in Table S2. * indicates significant differences in relative expression levels where the $p$ values of $t$-tests are less than -0.1 . 
Additional file 7: Table S3. List of MTEC specific genes that associated with mTECu-T-DMRs.

Additional file 8: Figure S5. Genes involved in the development of mTECs with mTEC-T-DMRs. White, mTECu-T-DMR; gray, mTECany2-T-DMR; black, no mTEC-T-DMR. The pathway was modified from Genome Network Platform (http://genomenetwork.nig.ac.jp). Tnfrsf1 1a (RANK) and its down-stream genes Traf2 and Lyn were associated with mTECany2-TDMRs and Traf5 was associated with mTECU-T-DMR. TANK (Tank), CIAP2 (Birc2) and Crinkled (Edaradd), which were recruited by TRAF2 to activate the downstream NF-KB [9-11]. Stimulation by RANK leads to activation of STAT1 which in turn up-regulates IFN-stimulated gene expression in mTECs [12]. Stat1 and Jak1 were found to be associated with mTECu-TDMR and mTECany2-T-DMR, respectively.

Additional file 9: Table S4. T-DMRs analyzed by COBRA

Additional file 10: Methods and references to additional files.

Additional file 11: Table S5. List of primers for bisulfite PCR.

\section{Abbreviations}

mTEC: Medullary thymic epithelial cells; cTEC: Cortical thymic epithelial cells; TRA: Tissue-restricted antigen; Aire: Autoimmune regulator; T-DMR: Tissuedependent and differentially methylated region; D-REAM: T-DMR profiling with restriction tag-mediated amplification; UEA1: Ulex europaeus agglutinin I.

\section{Competing interests}

The authors declare that they have no competing interests.

\section{Authors' contributions}

GW conceived the study, carried out experiments, analyzed the data, prepared the figures, and drafted the manuscript. $\mathrm{KH}$ performed the microarray experiment. SS assisted in bioinformatical analysis of D-REAM data. NA and TA assisted in isolating MTECs and control stromal cells. KS participated in the design of experiments. SY participated in the design of the experiments, performed bioinformatical analysis of D-REAM data, prepared the figures, and drafted the manuscript. All authors read and approved the final manuscript.

\section{Acknowledgements}

This research was supported by a Grant-in-Aid for Scientific Research from the Ministry of Education, Culture, Sports, Science and Technology (MEXT), Japan (no. 21221008 to K.S.); by a grant from the Japanese Society for the Promotion of Science (to TA); and by CREST from the Japan Science and Technology Agency (JST) (to SY).

\section{Author details}

'Laboratory of Cellular Biochemistry, Department of Animal Resource Sciences Neterinary Medical Science, Graduate School of Agricultural and Life Sciences, The University of Tokyo, 1-1-1, Yayoi, Bunkyo-ku, Tokyo 113-8657, Japan. ${ }^{2}$ Division of Cellular and Molecular Biology, Institute of Medical Science, The University of Tokyo, 4-6-1, Shirokane-dai, Minato-ku, Tokyo 108-8639, Japan.

Received: 23 June 2012 Accepted: 27 October 2012

Published: 2 November 2012

\section{References}

1. Kyewski B, Klein L: A central role for central tolerance. Annu Rev Immunol 2006, 24:571-606.

2. Mathis D, Benoist C: Aire. Annu Rev Immunol 2009, 27:287-312.

3. Derbinski J, Gäbler J, Brors B, Tierling S, Jonnakuty S, Hergenhahn M, Peltonen L, Walter J, Kyewski B: Promiscuous gene expression in thymic epithelial cells is regulated at multiple levels. J Exp Med 2005, 202(1):33-45.

4. Johnnidis JB, Venanzi ES, Taxman DJ, Ting JP, Benoist CO, Mathis DJ: Chromosomal clustering of genes controlled by the aire transcription factor. Proc Natl Acad Sci U S A 2005, 102(20):7233-7238.

5. Derbinski J, Pinto S, Rösch S, Hexel K, Kyewski B: Promiscuous gene expression patterns in single medullary thymic epithelial cells argue for a stochastic mechanism. Proc Natl Acad Sci U S A 2008, 105(2):657-662.
6. Gillard GO, Farr AG: Features of medullary thymic epithelium implicate postnatal development in maintaining epithelial heterogeneity and tissue-restricted antigen expression. J Immunol 2006, 176(10):5815-5824.

7. Dooley J, Erickson M, Farr AG: Alterations of the medullary epithelial compartment in the Aire-deficient thymus: implications for programs of thymic epithelial differentiation. J Immunol 2008, 181(8):5225-5232.

8. Senoo M, Pinto F, Crum CP, McKeon F: p63 Is essential for the proliferative potential of stem cells in stratified epithelia. Cell 2007, 129(3):523-536.

9. Yano M, Kuroda N, Han H, Meguro-Horike M, Nishikawa Y, Kiyonari H, Maemura K, Yanagawa Y, Obata K, Takahashi S, et al: Aire controls the differentiation program of thymic epithelial cells in the medulla for the establishment of self-tolerance. J Exp Med 2008, 205(12):2827-2838.

10. Matsumoto M: Contrasting models for the roles of Aire in the differentiation program of epithelial cells in the thymic medulla. Eur $J$ Immunol 2011, 41(1):12-17.

11. Hikosaka Y, Nitta T, Ohigashi I, Yano K, Ishimaru N, Hayashi Y, Matsumoto M, Matsuo K, Penninger JM, Takayanagi $H$, et al: The cytokine RANKL produced by positively selected thymocytes fosters medullary thymic epithelial cells that express autoimmune regulator. Immunity 2008, 29 (3):438-450

12. Akiyama T, Shimo Y, Yanai H, Qin J, Ohshima D, Maruyama Y, Asaumi Y, Kitazawa J, Takayanagi $\mathrm{H}$, Penninger JM, et al: The tumor necrosis factor family receptors RANK and CD40 cooperatively establish the thymic medullary microenvironment and self-tolerance. Immunity 2008, 29(3):423-437.

13. Shiota K, Kogo Y, Ohgane J, Imamura T, Urano A, Nishino K, Tanaka S, Hattori N: Epigenetic marks by DNA methylation specific to stem, germ and somatic cells in mice. Genes Cells 2002, 7(9):961-969.

14. Sakamoto H, Suzuki M, Abe T, Hosoyama T, Himeno E, Tanaka S, Greally JM, Hattori N, Yagi S, Shiota K: Cell type-specific methylation profiles occurring disproportionately in $\mathrm{CpG}$-less regions that delineate developmental similarity. Genes Cells 2007, 12(10):1123-1132.

15. Sakamoto H, Kogo Y, Ohgane J, Hattori N, Yagi S, Tanaka S, Shiota K: Sequential changes in genome-wide DNA methylation status during adipocyte differentiation. Biochem Biophys Res Commun 2008, 366(2):360-366.

16. Yagi S, Hirabayashi K, Sato S, Li W, Takahashi Y, Hirakawa T, Wu G, Hattori N, Ohgane J, Tanaka S, et al: DNA methylation profile of tissue-dependent and differentially methylated regions (T-DMRs) in mouse promoter regions demonstrating tissue-specific gene expression. Genome Res 2008, 18(12):1969-1978.

17. Sato S, Yagi S, Arai Y, Hirabayashi K, Hattori N, Iwatani M, Okita K, Ohgane J, Tanaka S, Wakayama T, et al: Genome-wide DNA methylation profile of tissue-dependent and differentially methylated regions (T-DMRs) residing in mouse pluripotent stem cells. Genes Cells 2010, 15(6):607-618

18. Kikuchi R, Yagi S, Kusuhara H, Imai S, Sugiyama Y, Shiota K: Genome-wide analysis of epigenetic signatures for kidney-specific transporters. Kidney Int 2010, 78(6):569-577.

19. Murumägi $A$, Vähämurto $P$, Peterson $P$ : Characterization of regulatory elements and methylation pattern of the autoimmune regulator (AIRE) promoter. J Biol Chem 2003, 278(22):19784-19790.

20. Kont V, Murumägi A, Tykocinski LO, Kinkel SA, Webster KE, Kisand K, Tserel L, Pihlap M, Ströbel P, Scott HS, et al: DNA methylation signatures of the AIRE promoter in thymic epithelial cells, thymomas and normal tissues. Mol Immunol 2011, 49(3):518-526.

21. Tykocinski LO, Sinemus A, Rezavandy E, Weiland Y, Baddeley D, Cremer C, Sonntag S, Willecke K, Derbinski J, Kyewski B: Epigenetic regulation of promiscuous gene expression in thymic medullary epithelial cells. Proc Natl Acad Sci U S A 2010, 107(45):19426-19431.

22. Anderson MS, Venanzi ES, Klein L, Chen Z, Berzins SP, Turley SJ, von Boehmer $\mathrm{H}$, Bronson R, Dierich A, Benoist C, et al: Projection of an immunological self shadow within the thymus by the aire protein. Science 2002, 298(5597):1395-1401.

23. Rodewald HR: Thymus organogenesis. Annu Rev Immunol 2008, 26:355-388.

24. Nishikawa Y, Hirota F, Yano M, Kitajima H, Miyazaki J, Kawamoto H, Mouri Y, Matsumoto M: Biphasic Aire expression in early embryos and in medullary thymic epithelial cells before end-stage terminal differentiation. J Exp Med 2010, 207(5):963-971.

25. Candi E, Rufini A, Terrinoni A, Giamboi-Miraglia A, Lena AM, Mantovani R, Knight R, Melino G: DeltaNp63 regulates thymic development through enhanced expression of FgfR2 and Jag2. Proc Natl Acad Sci U S A 2007, 104(29):11999-12004. 
26. Rossi SW, Kim MY, Leibbrandt A, Parnell SM, Jenkinson WE, Glanville SH, McConnell FM, Scott HS, Penninger JM, Jenkinson EJ, et al: RANK signals from CD4(+)3(-) inducer cells regulate development of Aire-expressing epithelial cells in the thymic medulla. J Exp Med 2007, 204(6):1267-1272.

27. Russell HI, York IA, Rock KL, Monaco JJ: Class II antigen processing defects in two $\mathrm{H} 2 \mathrm{~d}$ mouse cell lines are caused by point mutations in the $\mathrm{H} 2$ DMa gene. Eur J Immunol 1999, 29(3):905-911.

28. Zuklys S, Balciunaite G, Agarwal A, Fasler-Kan E, Palmer E, Holländer GA: Normal thymic architecture and negative selection are associated with Aire expression, the gene defective in the autoimmunepolyendocrinopathy-candidiasis-ectodermal dystrophy (APECED). $\mathrm{J}$ Immunol 2000, 165(4):1976-1983.

29. Van Vliet E, Jenkinson EJ, Kingston R, Owen JJ, Van Ewijk W: Stromal cell types in the developing thymus of the normal and nude mouse embryo. Eur J Immunol 1985, 15(7):675-681.

30. Gray DH, Chidgey AP, Boyd RL: Analysis of thymic stromal cell populations using flow cytometry. J Immunol Methods 2002, 260(1-2): $15-28$.

31. Johnson WE, Li W, Meyer CA, Gottardo R, Carroll JS, Brown M, Liu XS: Model-based analysis of tiling-arrays for ChIP-chip. Proc Natl Acad Sci U S A 2006, 103(33):12457-12462.

32. Goecks J, Nekrutenko A, Taylor J, Team G: Galaxy: a comprehensive approach for supporting accessible, reproducible, and transparent computational research in the life sciences. Genome Biol 2010, 11(8):R86.

33. Ihaka R, Gentleman R: $R$ : a language for data analysis and graphics. J. Comp. Graph. Stat 1996, 5(3):299-314.

34. Edgar R, Domrachev M, Lash AE: Gene Expression Omnibus: NCBI gene expression and hybridization array data repository. Nucleic Acids Res 2002, 30(1):207-210.

35. Wu ZJ, Irizarry RA, Gentleman R, Martinez-Murillo F, Spencer R: A modelbased background adjustment for oligonucleotide expression arrays. J Am Stat Assoc 2004, 99:909-917.

36. Haider S, Ballester B, Smedley D, Zhang J, Rice P, Kasprzyk A: BioMart Central Portal-unified access to biological data. Nucleic Acids Res 2009, 37(Web Server issue):W23-W27.

doi:10.1186/1471-2172-13-58

Cite this article as: Wu et al:: DNA methylation profile of Aire-deficient mouse medullary thymic epithelial cells. BMC Immunology 2012 13:58.

\section{Submit your next manuscript to BioMed Central and take full advantage of:}

- Convenient online submission

- Thorough peer review

- No space constraints or color figure charges

- Immediate publication on acceptance

- Inclusion in PubMed, CAS, Scopus and Google Scholar

- Research which is freely available for redistribution 